\title{
Effect of postmenopausal strontium ranelate treatment on oxidative stress in rat skin tissue
}

\author{
Mehmet Berköz ${ }^{1^{\star}}$, Özgün Sağır ${ }^{2}$, Serap Yalın², Ülkü Çömelekoğlu ${ }^{3}$, Fatma Söğüt ${ }^{4}$, Pelin Eroğlu ${ }^{5}$ \\ ${ }^{1}$ Department of Pharmaceutical Biotechnology, Faculty of Pharmacy, V an Y uzuncu Yil University, Van, Turkey \\ ${ }^{2}$ Department of Biochemistry, Faculty of Pharmacy, Mersin University, Mersin, Turkey \\ ${ }^{3}$ Department of Biophysics, Faculty of Medicine, Mersin University, Mersin, Turkey \\ ${ }^{4}$ Department of Medical Services and Techniques, Vocational School of Health Services, Mersin University, Mersin, \\ Turkey \\ ${ }^{5}$ Department of Chemistry, Faculty of Science and Literature, Mersin University, Mersin, Turkey
}

\begin{abstract}
Strontium ranelate is one of the most common post-menopausal osteoporosis medications that have been used in recent years. The effects of strontium ranelate treatment on the free radical-antioxidant balance of the skin have not been fully elucidated. In this study, we investigated the effects of strontium ranelate treatment on oxidative stress parameters in the skin tissue of rats with the experimental osteoporosis model. Total 35 rats were divided to five groups as control group (Group I), oophorectomy group (Group II), strontium ranelate group (Group III), oophorectomy + strontium ranelate group (immediately after the oophorectomy) (Group IV) and oophorectomy + strontium ranelat group (after three months of oophorectomy) (Group V). In Group III, IV and V, $500 \mathrm{mg} / \mathrm{kg}$ doses of strontium were given to rats by orally. At the end of the study, superoxide dismutase (SOD) and catalase activities, malondialdehyde (MDA) and glutathione (GSH) levels of the rats' skin tissues were determined. Catalase and SOD activities and GSH levels of all groups were significantly lower than Group I ( $\mathrm{p}<0.05)$. MDA levels of all groups were significantly higher than Group I $(\mathrm{p}<0.05)$. Catalase and SOD activities and GSH levels of Group IV and V were significantly lower than Group II ( $<<0.05)$. MDA levels of Group IV and $\mathrm{V}$ were significantly higher than Group II $(\mathrm{p}<0.05)$. In conclusion, strontium ranelate treatment in oophorectomized rats has resulted in an increase in oxidative stress in skin tissue and can cause to several skin disorders.
\end{abstract}

Key Words: Skin, strontium ranelate, osteoporosis, oxidative stress, antioxidant

\section{Introduction}

Osteoporosis is a common chronic illness that may result in osteoporotic fractures of major bones and occurs in more than $20 \%$ of women and $5 \%$ of men age 50 and older. Bone is a dynamic tissue that is continuously being remodeled as old bone is removed and new bone is formed. This process of bone turnover maintains mineral homeostasis, skeletal size and mechanical strength, and provides a mechanism by which damaged bone can be repaired. Bone turnover is balanced in healthy young adults, with as much bone being formed as is being reabsorbed. However, during menopause, the process of bone turnover increases and becomes uncoupled, resulting in a faster rate of bone resorption which cannot be compensated for by bone formation, resulting in an overall loss of bone (1). Sex hormone deficiency in postmenopause term in women and andropause term in men, suboptimal calcium and vitamin D intake or malnutrition and absance of exercise are the most seen osteoporosis etiologic causes (2). Drugs for osteoporosis can be classified as two major classes: anabolic agents and resorption inhibitors. While anabolic agents stimulate osteoblastic differentiation and activity, resorption inhibitors depress bone resorption on osteoclasts. In the treatment of osteoporosis, bone remodeling medications such as calcium, hormone replacement therapy (especially estrogen), vitamin $\mathrm{D}$ and active metabolites, biphosphates and calcitonin, fluoride, anabolic steroids (especially testosterone), parathyroid hormone and peptides, growth hormone and ipriflavone drugs can also be used. One of the most common post-menopausal osteoporosis medications that have been used in recent years is strontium ranelate (3).

Strontium ranelate, also called SI 2911, has been obtained from two stable non-radioactive strontium atoms and ranelic acid compound and has been licensed for postmenopausal osteoporosis treatment. Strontium ranelate has a double effect on bone metabolism; inducing the osteogenesis, osteoblastic cell replication and 
activity and reducing the preosteoclast differentiation, osteoclastic activity and resorption of bones in a dose-dependent manner (4). During strontium ranelate therapy, some side effects, such as, headache, diarrhea, nausea, vomiting, abdominal pain, fever, swollen glands, mouth ulcers, skin rash, dermatitis, eczema, pruritus, urticaria, angioederma and Stevens - Johnson syndrome can be seen. These data indicate that strontium ranelate treatment can trigger skin disorders. One of the most important causes of skin disorders is the impairment of the antioxidant-free radical balance of the skin because of the physiological and pathological conditions (5). However, the effects of strontium ranelate treatment on the free radical-antioxidant balance of the skin tissue have not been fully elucidated. In this study, we investigated the effects of strontium ranelate treatment on oxidative stress parameters in the skin tissue of rats with the experimental menopause rat model.

\section{Material and methods}

Animals: 90 days old 35 female Wistar-Albino rats weighing $223 \pm 21 \mathrm{~g}$ were used in our study. Rats were matched to laboratory conditions one week prior and feed and water consumption was not restricted. Rats were housed in a room with a temperature of $23 \pm 2$ ${ }^{\circ} \mathrm{C}$ and a humidity of $55 \pm 10 \%$ throughout the experiment. The ventilation of the laboratory where the animals were housed was provided by means of a window type aspirator and the 12 hour light to 12 hour darkness was observed for illumination. Rats were given medication every morning between $09^{00}$ $10^{00} \mathrm{am}$. Rats were not fed for 12 hours prior to sacrifice. The ethical approval of the study was taken from Animal Experiments Ethics Committee of the Mersin University.

Experimental Groups: Our study was designed in 5 groups as 7 animals in each group. These groups are;

Group I (Control group): Animals in this group were sacrificed three months after the beginning of the study without any treatment.

Group II (Oophorectomy group): In this group, animals were oophorectomized at the beginning of the study and at the end of this study; all the animals in this group were sacrificed.

Group III (Strontium ranelate group): Animals in this group received $500 \mathrm{mg} / \mathrm{kg}$ strontium ranelate daily for three months and were sacrificed at the end of the third month (6).

Group IV (Oophorectomy + Strontium ranelate group (immediately after the oophorectomy)): At the beginning of study, the animals were oophorectomized and a daily $500 \mathrm{mg} / \mathrm{kg}$ strontium ranelate was applied for three months. At the end of this study, all the animals were sacrificed.

Group V (Oophorectomy + Strontium ranelat group (after three months of overectomy)): Three months after the oophorectomy, animals were treated with 500 $\mathrm{mg} / \mathrm{kg}$ strontium ranelate daily for three months and then, all the animals were sacrificed.

Surgical procedures: All rats undergoing to oophorectomy were anesthetized with $200 \mathrm{mg} / \mathrm{kg} / \mathrm{i} . \mathrm{p}$. ketamine $\mathrm{HCl}$ and $10 \mathrm{mg} / \mathrm{kg} /$ i.p. xylazine. The fur in the operation area of the female rats was cleared and the area was cleared with batticon. The abdominal cavities of the rats were opened, both the right and left fallopian tubes were found, the proximal and distal ends of the tubes were cleaved and the ovaries were isolated. The exposed tuba uterine ends were ligated with '0000 chrome catgut'. After the operation, the abdominal cavity and skin texture were sewn and dressed with batticon to prevent the possible infections. All surgical materials used during the operation were sterilized before and after the operation (7). The operation was carried out at Mersin University Experimental Research Center.

Tissue Preparation: All animals that completed the experimental period were anesthetized with ketamine and xylazine. Sacrification of the anesthetized rats was performed by cardiac puncture. Skin tissues of all rats were isolated and the tissues were cleaned by passing through $0.9 \% \mathrm{NaCl}$ solution. Until the study day, all tissues were stored in deep freezing $\left(-70^{\circ} \mathrm{C}\right)$.

On the day of the study, $0.1 \mathrm{~g}$ of skin tissue sample was taken from the deep-freeze-dried skin tissue samples and $1900 \mu \mathrm{l}$ of cold phosphate buffer (PBS). All tissues were homogenized in a cold jacket for 3 minutes at $16,000 \mathrm{rpm}$. The homogenate was transferred to the tubes without increasing the heat and the tubes were numbered. The homogenates were centrifuged and the supernatants were used for lipid peroxidation determination and antioxidant enzyme assays.

\section{Biochemical assays}

Malondialdehyde (MDA) Level: MDA reacts with thiobarbituric acid (TBA), giving a spectroscopically readable final product at $532 \mathrm{~nm}$ (8).

Superoxide dismutase (SOD) Activity: The principle of measurement of SOD enzyme activity, which accelerates the aquatic and molecular oxygen dismutation of endogenous and exogenous sources of toxic superoxide radicals generated during the production of oxidative pathway energy, is based on the spectrophotometric measurement of superoxide radicals which are released by xanthine oxidase in the 
presence of xanthine in the presence of nitroblue tetrazolium (NBT) at $560 \mathrm{~nm}$ according to Sun et al (9).

Catalase Activity: The activity of the enzyme catalase was analysed according to Aebi method (10), measuring the initial rate of $\mathrm{H}_{2} \mathrm{O}_{2}$ decomposition at $240 \mathrm{~nm}$.

Reduced Glutathione (GSH) level: GSH analysis was performed according to the method reported by Beutler et al (11). In this method, all proteins that do not carry the sulfhydryl in the tissue homogenates are precipitated. In the obtained clear liquid, the yellow complex formed by 5,5'-Dithiobis(2-nitrobenzoic acid) (DTNB) and sulfhydryl groups is measured colorimetrically at a wavelength of $412 \mathrm{~nm}$.

The protein concentration in the supernatant was estimated by Lowry et all's method (12).

Statistical analysis: SPSS 15.0 statistical program was used for all statistical evaluations. One way analysis of variance test (ANOVA) with Tukey's posthoc test was used to determine statistical difference between groups. Reported data were presented as mean \pm standard deviation and values having $\mathrm{p}$ value $<0.05$ were considered to be significant.

\section{Results}

Catalase and SOD activities and GSH levels of all groups were significantly lower than Group I $(p<0.05)$. MDA levels of all groups were significantly higher than Group I $(p<0.001)$. Catalase and SOD activities and GSH levels of Group IV and $\mathrm{V}$ were significantly lower than Group II $(p<0.05)$. MDA levels of Group IV and V were significantly higher than Group II $(p<0.001)$. No statistically significant difference was found between Group II and Group III on any of the parameters examined. All data were shown in Table 1.

\section{Discussion}

Osteoporosis affects approximately two hundred million individuals in all over the world. It is estimated $50 \%$ of women over the age of 50 , as well as one in four men will fracture a bone due to osteoporosis. Major risk factors for osteoporosis include aging and oxidative stress. Oxidative stress is caused by the cumulative formation of oxygenderived damaging free radicals that are inadequately neutralized by antioxidants and can be linked to the aging process. Oxidative stress is involved in osteoclastogenesis that leads to bone resorption by osteoclasts, the catabolic cells of bone tissue. When the action of bone resorption exceeds that of bone formation, osteoporosis or thinning of the bones will occur. Several studies have been published that support the use of the oophorectomized rat model for the study of osteoporosis (13).

In both age-related osteoporosis in human and experimental menopause model in laboratory animals, an increase in oxidative stress is observed due to increased lipid peroxidation and decreased antioxidant defense system. In addition, the development of osteoporosis due to increased lipid peroxidation is also a paradox $(14,15)$. Sanchez-Rodriguez et al (16)

Table 1. MDA and GSH levels and SOD and catalase activities in rat skin tissue

\begin{tabular}{lccccc}
\hline & $\begin{array}{c}\text { Group I } \\
(\mathrm{n}=7)\end{array}$ & $\begin{array}{c}\text { Group II } \\
(\mathrm{n}=7)\end{array}$ & $\begin{array}{c}\text { Group III } \\
(\mathrm{n}=7)\end{array}$ & $\begin{array}{c}\text { Group IV } \\
(\mathrm{n}=7)\end{array}$ & $\begin{array}{c}\text { Group V } \\
(\mathrm{n}=7)\end{array}$ \\
\hline $\begin{array}{l}\text { MDA level } \\
\text { (nmol/mg prt. })\end{array}$ & $70.48 \pm 9.28$ & $144.8 \pm 19.26^{\mathrm{a}}$ & $139.56 \pm 25.84^{\mathrm{a}}$ & $215.94 \pm 27.54^{\mathrm{a}, \mathrm{b}}$ & $223.09 \pm 31.25^{\mathrm{a}, \mathrm{b}}$ \\
\hline $\begin{array}{l}\text { GSH level } \\
\text { (nmol/mg prt. })\end{array}$ & $0.143 \pm 0.021$ & $0.091 \pm 0.012^{\mathrm{c}}$ & $0.085 \pm 0.013^{\mathrm{c}}$ & $0.073 \pm 0.014^{\mathrm{c}}$ & $0.074 \pm 0.018^{\mathrm{c}}$ \\
\hline $\begin{array}{l}\text { SOD activity } \\
\text { (U/mg prt.) }\end{array}$ & $10.06 \pm 1.4$ & $3.57 \pm 1.44^{\mathrm{c}}$ & $5.23 \pm 2.19 \mathrm{c}$ & $2.02 \pm 0.18^{\mathrm{c}, \mathrm{d}}$ & $2.06 \pm 0.33^{\mathrm{c}, \mathrm{d}}$ \\
\hline $\begin{array}{l}\text { Catalase activity } \\
\text { (U/mg prt.) }\end{array}$ & $44.76 \pm 3.19$ & $43.01 \pm 5.26$ & $42.88 \pm 9.04^{\mathrm{e}}$ & $36.29 \pm 5.47^{\mathrm{e}, \mathrm{f}}$ & $33.94 \pm 7.63^{\mathrm{e}, \mathrm{f}}$ \\
\hline
\end{tabular}

Values are expressed as mean \pm S.D.

a Significantly higher comparing with Group I $(\mathrm{p}<0.001)$

b Significantly higher comparing with Group II $(\mathrm{p}<0.001)$

c Significantly lower comparing with Group I $(\mathrm{p}<0.001)$

d Significantly lower comparing with Group II $(\mathrm{p}<0.001)$

e Significantly lower comparing with Group I $(\mathrm{p}<0.05)$

f Significantly lower comparing with Group II $(\mathrm{p}<0.05)$ 
determined that the ratio of SOD/GSH-Px was significantly higher in osteoporotic women than that was seen in healthy controls and was negatively correlated with BMD. They postulate that this imbalance between GSH-Px and SOD is due to increased concentrations of hydrogene peroxide $\left(\mathrm{H}_{2} \mathrm{O}_{2}\right)$, resulting in elevated oxidative stress in participants with osteoporosis (16). Prasad et al (17) demonstrated that in fracture patients total antioxidant capacity is significantly elevated one to two weeks following fracture compared to baseline values and healthy controls. These findings suggest that antioxidant capacity is elevated post-fracture as a compensatory mechanism attempt to repair the damage caused by oxidative stress. This defense mechanism may be overwhelmed while attempting to quench the increased superoxide radical $\left(\mathrm{O}_{2}^{-}\right)$ production by osteoclasts during fracture healing, as is evidenced later by significantly depressed SOD and GSH-Px (17).

In menopausal women, skin is one of the most affected organs (18). In this study, we aimed to investigate the effect of strontium ranelate as an osteoblastic activity enhancing agent on the oxidative stress in the skin tissue in oophorectomized female rats. As a result of the oophorectomy, there was a statistically significant increase in the MDA level in the skin tissue of rats and a significant decrease in GSH level and in SOD activity, but no change in catalase activity. The reason for this is probably due to the excessive consumption of SOD enzyme to cleanse the superoxide in the skin tissue after the oophorectomy.

The use of strontium salts in postmenopausal osteoporosis treatment has gained importance in recent years. The most important of these are strontium chloride and strontium ranelate. Strontium, a bivalent alkaline earth metal, is similar to calcium and barium according to its chemical properties (4). Heavy metals and several non-metals have many negative effects on antioxidant defense system. Subchronic lead acetate exposure has been shown to induce oxidative stress in the rat brain tissue and lead to brain damage. Radical oxygen species and radical production are shown as a mechanism for acute cadmium toxicity. Arsenic can lead to oxidative stress in the cerebellum and cerebral hemispheres by increasing lipid peroxidation and decreasing glutathione level, SOD and catalase activities. Toxicity of selenium compounds is due to prooxidant catalytic activities for $\mathrm{H}_{2} \mathrm{O}_{2}, \mathrm{O}_{2}-$ and similar oxy radical production. In addition, the oxidation of glutathione by selenite produces $\mathrm{O}_{2}^{-\cdot}(19,20)$. When we applied strontium ranelate as a therapeutic agent in our study to non-oophorectomized rats, there was an increase in skin MDA level, a decrease in GSH level, and a decrease in SOD activity compared to the control group, but there was no change in catalase activity. We think that such a result may be caused by the toxic effect of the strontium ranelate.

There are many studies in the literature that examine the effects of many drugs used in the treatment of osteoporosis on the oxidative stress resulting from this disease (7). Yalin et al (21) found a statistically significant decrease in liver MDA level, SOD and catalase activities when compared with the control group when treated the oophorectomized rats with alendronate. In the same study, when risedronate was administered to the oophorectomized rats, there was a significant decrease in MDA level compared to the control group. We think that this increase in SOD and catalase activities due to eliminate the ROS forming in the organism (21). We also think that risendronate administration results in a statistically significant decrease in the level of MDA, which is due to the fact that this drug treats osteoporosis without triggering the oxidative stress. In a study conducted by Berkoz et al (22), it was found that administration of calcitonin to oophorectomized rats increased MDA levels in liver and kidney tissues and decreased liver catalase activity. In our study, we observed that strontium ranelate administration as a prophylactic (group IV) or therapeutic (group V) increased MDA levels in rat skin tissue, increased GSH level, SOD and catalase activities in oophorectomized rats compared to the control group. Prophylactic or therapeutic application of strontium ranelate to oophorectomized rats did not lead to a statistically significant difference in the parameters that we examined.

We also compared the oxidative stress parameters of the oophorectomized rats treated with prophylactic or therapeutic strontium ranelate with the only oophorectomized rats. Our results showed that administration of prophylactic or therapeutic strontium ranelate application to the oophorectomized rats increased MDA levels, decreased SOD and catalase activities, and did not alter GSH levels compared to the oophorectomized rats in the skin tissue. In literature, Berköz et al (22) found that liver and kidney MDA levels increased and liver catalase activity decreased after the administration of calcitonin to oophorectomized rats. Yalin et al (21) observed that risendronate administration reduced liver MDA levels and increased catalase activity in oophorectomized rats, nevertheless alendronate administration increased MDA levels, SOD and catalase activities in liver tissue of the oophorectomized rats. In our study it was suggested that this dramatic increase in the oxidative stress parameters as a result of pro-oxidant effects of strontium ranelate treatment after the 
postmenopausal osteoporosis, similar to the other anti-osteoporotic agents.

In conclusion, antioxidant levels in the skin tissue of oophorectomized rats were found to be low and this may increase the oxidative stress and may be a risk factor in terms of photo-aging, skin cancer, atopic dermatitis, pigmentation disorders and other skin diseases. Strontium ranelate treatment also reduces the antioxidant levels in the skin tissue and can lead to dramatic consequences by further increasing the oxidative stress induced by menopause. The use of strontium ranelate treatment both as prophylactic or therapeutic purpose has resulted in a decrease in the antioxidant capacity, an increase in lipid peroxidation and an increase in oxidative stress. The increase in oxidative stress was more dramatic in prophylactic strontium ranelate treatment group. This suggests that the treatment of strontium ranelate after oophorectomy increased oxidative stress. Therefore, the patients treated with strontium ranelate should be measured for their serum antioxidant levels at certain intervals and if there is a significant decrease in enzyme activity, it can be necessary to terminate the treatment by considering the profit-loss balance or if it is absolutely necessary to continue to the treatment, the dosage of the drug should be decreased and antioxidant supplementation should be provided to the patient when it is necessary, so that the photoaging and other skin diseases which may develop due to the oxidative stress may be avoided.

\section{References}

1. Ensrud KE, Crandall CJ. Osteoporosis. Ann Intern Med 2017; 16: 17-32.

2. Duque G, Daly RM, Sanders K, Kiel DP. Vitamin D, bones and muscle: myth versus reality. Australas J Ageing 2017; 36: 8-13.

3. Martínez-Morillo M, Grados D, Holgado S. Premenopausal osteoporosis: how to treat? Reumatol Clin 2012; 8: 93-97.

4. Canalis E, Hott M, Deloffre P, Tsouderos Y, Marie PJ. The divalent salt S12911 enhances bone cell replication and bone formation in vitro. Bone 1996; 18: 517-523.

5. Rossini M, Adami G, Adami S, Viapiana O, Gatti D. Safety issues and adverse reactions with osteoporosis management. Expert Opin Drug Saf 2016; 15: 321-332.

6. Chen B, Li Y, Yang X, Xie D. Comparable effects of alendronate and strontium ranelate on femur in ovariectomized rats. Calcif Tissue Int 2013; 93: 481-486.
7. Hatungil R, Yalın S, Çömelekoğlu Ü, et al. Cadmium induced changes in lipid peroxidation and antioxidant status in brain of ovariectomized rats. Asian J Chem 2006; 18: 1467-1473.

8. Yagi K. Simple procedure for specific enzyme of lipid hydroperoxides in serum or plasma. Methods Mol Biol 1998; 108: 107-110.

9. Sun Y, Oberley LW, Ying L. A simple method for clinical assay of superoxide dismutase. Clin Chem 1988; 34: 497-500.

10. Aebi H. Catalase in vitro. Methods Enzymol 1984; 105: 121-126.

11. Beutler E, Duron O, Kelly BM. Improved method for the determination of blood glutathione. J Lab Clin Med 1963; 61: 882-888.

12. Lowry OH, Rosebrough NJ, Farr AL, Randall RJ. Protein measurement with the Folin phenol reagent. J Biol Chem 1961; 193: 265-275.

13. da Palma RK, Moraes-Silva IC, da Silva Dias D, et al. Resistance or aerobic training decreases blood pressure and improves cardiovascular autonomic control and oxidative stress in hypertensive menopausal rats. J Appl Physiol 2016; 121: 10321038.

14. Spilmont M, Léotoing L, Davicco MJ, et al. Pomegranate peel extract prevents bone loss in a preclinical model of osteoporosis and stimulates osteoblastic differentiation in vitro. Nutrients 2015; 7: 9265-9284.

15. Hahn M, Conterato GM, Frizzo CP, et al. Effects of bone disease and calcium supplementation on antioxidant enzymes in postmenopausal women. Clin Biochem 2008; 41: 69-74.

16. Sánchez-Rodríguez MA, Ruiz-Ramos M, CorreaMuñoz E, Mendoza-Núñez VM. Oxidative stress as a risk factor for osteoporosis in elderly Mexicans as characterized by antioxidant enzymes. BMC Musculoskelet Disord 2007; 8: 124-130.

17. Prasad N, Sunderamoorthy D, Martin J, Murray JM. Secondary prevention of fragility fractures: are we following the guidelines? Closing the audit loop. Ann R Coll Surg Engl 2006; 88: 470-474.

18. Duarte GV, Trigo AC, Paim de Oliveira Mde F. Skin disorders during menopause. Cutis 2016; 97 : 16-23.

19. Koedrith P, Seo YR. Advances in carcinogenic metal toxicity and potential molecular markers. Int J Mol Sci 2011; 12: 9576-9595.

20. Jomova K, Valko M. Advances in metalinduced oxidative stress and human disease. Toxicology 2011; 283: 65-87.

21. Yalın S, Comelekoglu U, Bagis S, Berkoz M, Eroglu P. Effects of alendronate and risedronate therapy on antioxidant enzyme activity and lipid peroxidation in ovariectomized rats. Turkish J Med Sci 2010; 40: 613-618.

22. Berkoz M, Yalın S, Comelekoglu U, Bagis S. Effect of calcitonin on lipid peroxidation in ovariectomized rats. Eur J Chem 2010; 1: 44-46. 